\title{
Editorial
}

\section{Still Comparing Apples With Oranges?}

\section{Some Thoughts on the Principles and Practices of Measurement Invariance Testing}

\author{
Samuel Greiff ${ }^{1}$ and Ronny Scherer ${ }^{2}$ \\ ${ }^{1}$ Institute of Cognitive Science and Assessment (COSA), University of Luxembourg, Luxembourg \\ ${ }^{2}$ Department of Teacher Education and School Research (ILS), Faculty of Educational Sciences, University of Oslo, Norway
}

Measurement invariance testing has become an integral part of the methodological repertoire in psychological assessment. Whenever researchers attempt to compare scales or test scores across groups or over time, it needs to be ensured that they do not compare "apples with oranges." Measurement invariance testing is the road that leads to the information about whether the comparisons of means or variances are valid. In other words, invariance testing examines the between-group or between-time points variation in factor means and variances by ruling out differences in measurement (Bauer, 2017). This ensures the fairness of scales or tests and therefore contributes to crafting a validity argument (Kane, 2013).

In this editorial, we briefly review some of the principles and practices of measurement invariance testing in psychological assessment contexts and encourage EJPA authors to critically review the limitations of the commonly used approach to invariance testing - multi-group confirmatory factor analysis. We suggest considering alternatives to this approach, for instance, when submitting to EJPA and we discuss the potential of these alternative approaches.

\section{Same Procedure as Every Time: Multi-Group Confirmatory Factor Analysis}

Measurement invariance across groups or over time is commonly tested by means of multi-group confirmatory factor analysis (CFA) - a procedure that compares factor models with different sets of equality constraints on parameters (Meredith, 1993). Indeed, our review of EJPA papers that performed measurement invariance testing showed that multi-group CFA was the method of choice in more than $70 \%$ of the studies (publication period: January 1995August 2017; for details, please refer to the Electronic Supplementary Material, ESM 1). Typically, once it has been established that the overall setup of a measurement model is comparable across groups or over time (configural invariance), equality constraints of the factor loadings, item intercepts, and residual variances are introduced, leading up to metric, scalar, and strict invariance. However, the list of available parameter constraints and combinations thereof can be extended in order to meet other levels of invariance (see, for example, Marsh et al., 2009).

To identify which level of invariance is met, multi-group models with different constraints are compared against each other by means of chi-square difference testing or by evaluating differences in fit indices, such as the CFI, RMSEA, or SRMR, against suggested cut-offs. At this point, we would like to emphasize that these cut-offs must not be considered "golden rules" (see also Greiff \& Heene, 2017) they do not generalize to all contexts (e.g., data structures and types) and thus require a critical evaluation of the contexts in which they are applied. Among others, these cut-offs depend on the treatment of item responses (i.e., continuous vs. categorical), the number of factors, the type of measurement model (e.g., correlated-factors vs. nestedfactor models), the number of groups or time points, and the level of invariance that is tested (e.g., Khojasteh \& Lo, 2015; Rutkowski \& Svetina, 2014). Besides the issues related to the sensitivity of model fit indices toward detecting measurement invariance, multi-group CFA bears a number of other limitations with regard to factor structure and the scale of the grouping variable, which has to be discrete. For complex psychological measures, however, more complex factor structures may occur; in addition, in some research contexts, invariance across continuous rather than 
categorical variables (e.g., age) is of interest. In the following, we will bring to attention alternatives to multi-group CFA that address these issues.

\section{And Yes, There Is More: Procedures Beyond Multi-Group CFA}

Researchers often find themselves in situations where the desired level of measurement invariance, especially scalar invariance to compare factor means across groups, is not achieved by means of multi-group CFA. Marsh et al. (2017) even argue that some levels of invariance are "an unachievable ideal that in practice can only be approximated" (p. 1). Hence, the question is: What are the options researchers have if measurement invariance testing by multi-group CFA fails or if they want to explore other options in general?

\section{Non-Invariance Is a Finding}

We consider evidence against invariance a relevant finding, especially when presenting newly developed measures or well-established measures that have been studied in new contexts or for new samples without establishing measurement invariance. Knowledge on non-invariance can aid the creation of a validity argument and informs test developers and researchers about the functioning of the scale or test (Kane, 2013). In this sense, we encourage EJPA authors to not shy away from reporting negative results concerning the invariance of certain measures. However, we also encourage authors to take the evaluation of invariance a step further, either by discussing possible reasons for departures from measurement invariance and the consequences for the interpretation of the scale or test scores or by identifying statistically the items or subsets of items that function differently across groups or over time. To accomplish the latter, existing approaches to differential item functioning (DIF), such as the multiple indicators multiple causes (MIMIC) models or models based on item response theory, are particularly helpful and can supplement the findings obtained from multi-group CFA (e.g., Hatlevik, Scherer, \& Christophersen, 2017; Woods, 2009). Alternatively, authors may relax some of the parameter constraints in multi-group CFA invariance models and thus establish levels of partial measurement invariance. Reporting partial invariance, however, needs a thorough documentation of the steps authors have taken to arrive at these levels and might be less preferable for EJPA.

\section{Alternatives to Multi-Group CFA}

Over the last decade, several approaches to measurement invariance testing have been presented in the psychometric literature, some of which address the limitations of multigroup CFA directly and thus provide viable alternatives to the standard approach. Table 1 shows a selection of these approaches.

In some instances, the factor structure of a scale or test is best represented by complex rather than simple structures. In other words, more than one factor may explain variation in the responses to one item. Besides nested factor models, exploratory structural equation models provide viable alternatives to CFA that account for these aberrancies from simple structures. Marsh et al. (2009) extended the exploratory SEM framework to multi-group models for which measurement invariance can be tested directly. Another class of models that accounts for possible cross-loadings in factor structures refers to Bayesian structural equation models. Bayesian SEM uses priors and prior distributions of parameters in factor models rather than point estimates - a feature that allows including cross-loadings in multi-group models. Besides, instead of establishing exact invariance, approximate invariance can be established based on approximate equality constraints of model parameters across groups or over time (Van De Schoot et al., 2013).

Another issue that often leads to non-invariance based on multi-group CFA concerns the number of groups or time points for which invariance is tested - the more groups or time points, the less likely it is to find higher levels of invariance, especially scalar invariance (Marsh et al., 2017). Recently, two approaches to invariance across many groups have received attention: the alignment method and multilevel factor analysis (Muthén \& Asparouhov, 2017). The former estimates group-specific factor means and variances to approximate scalar invariance. The latter quantifies between-group variation in model parameters (e.g., factor loadings, item intercepts) using random effects and allows researchers to detect possible sources of non-invariance (Kim, Cao, Wang, \& Nguyen, 2017).

In some instances, researchers may want to test measurement invariance across originally continuous variables, such as age or socioeconomic status. Instead of arbitrarily categorizing these variables for multi-group CFA, researchers may well treat them continuously and perform a more direct and flexible invariance testing procedure - moderated factor analysis (Bauer, 2017). This procedure describes variation in model parameters (e.g., factor loadings, item intercepts, factor means and variances) as a function of continuous and/or categorical variables.

Besides, even more sophisticated approaches exist and researchers should make use of them. Brandmaier, von Oertzen, McArdle, and Lindenberger (2013) presented structural equation modeling trees - a data-mining procedure that identifies previously unobserved groups in the data set for which parameters in a structural equation model differ. Kim et al. (2017) argued that factor mixture 
Table 1. Selected measurement invariance testing procedures in multi-group settings

\begin{tabular}{|c|c|c|c|}
\hline Procedure & Description & Grouping variable & References \\
\hline Multi-group CFA & $\begin{array}{l}\text { Testing a series of equality constraints on } \\
\text { parameters in measurement models that } \\
\text { have a simple or nested structure across } \\
\text { groups }\end{array}$ & Categorical & $\begin{array}{l}\text { Pendergast, von der Embse, Kilgus, } \\
\text { and Eklund (2017) }\end{array}$ \\
\hline Multi-group Exploratory SEM & $\begin{array}{l}\text { Testing a series of equality constraints on } \\
\text { parameters in measurement models that } \\
\text { have a complex structure across groups }\end{array}$ & Categorical & Marsh, Morin, Parker, and Kaur (2014) \\
\hline Multi-group Bayesian SEM & $\begin{array}{l}\text { Testing the sensitivity of approximate } \\
\text { equality constraints on model parameters } \\
\text { across groups }\end{array}$ & Categorical & Van De Schoot et al. (2013) \\
\hline Alignment method & $\begin{array}{l}\text { Estimation of group-specific factor means } \\
\text { and variances to approximate scalar } \\
\text { invariance }\end{array}$ & Categorical & $\begin{array}{l}\text { Marsh et al. (2017); Muthén } \\
\text { and Asparouhov (2017) }\end{array}$ \\
\hline Multilevel factor analysis & $\begin{array}{l}\text { Testing a series of equality constraints on } \\
\text { parameters in measurement models } \\
\text { across groups - the grouping variable is } \\
\text { considered random }\end{array}$ & Categorical & Muthén and Asparouhov (2017) \\
\hline Moderated factor analysis & $\begin{array}{l}\text { Testing moderation effects of grouping } \\
\text { variables and/or covariates on item } \\
\text { parameters (i.e., tests for differential item } \\
\text { functioning) }\end{array}$ & $\begin{array}{l}\text { Categorical } \\
\text { and/or } \\
\text { continuous }\end{array}$ & Bauer (2017) \\
\hline
\end{tabular}

Note. CFA = Confirmatory factor analysis, SEM = Structural equation modeling.

models may also be used to identify unobserved groups under certain invariance constraints. Finally, Hildebrandt, Lüdtke, Robitzsch, Sommer, and Wilhelm (2016) proposed locally weighted structural equation modeling to examine measurement invariance across continuous variables (e.g., age), next to moderated factor analysis.

\section{Conclusion}

Overall, this editorial sought to encourage EJPA authors to take a critical approach to the use of multi-group CFA for invariance testing. We would like to highlight three aspects surrounding the principles and practices of invariance testing via multi-group CFA: First, the application of model fit criteria to compare nested invariance models should always be evaluated considering the complexity of measurement models, the study sample, and the treatment of data - the standard model fit criteria do not represent "golden rules". Second, standard invariance testing procedures, including multi-group CFA, have certain limitations - we therefore encourage authors to consider alternative, perhaps more suitable approaches to modeling their data. Third, we believe that the analysis of differential item functioning provides more specific information about whether certain items in a scale or test are invariant across groups or over time. This procedure can supplement more global tests of measurement invariance and thus strengthen the creation of a validity argument.

\section{Electronic Supplementary Material}

We have put together a supplementary material that provides a systematic overview of the current practices of measurement invariance testing employed in EJPA publications. We believe that this material provides a valuable source for authors who would like to (a) examine whether evidence for the invariance of certain scales has been reported in EJPA papers, and (b) explore the diversity of invariance testing approaches.

The electronic supplementary material is available with the online version of the article at https://doi.org/ $10.1027 / 1015-5759 / \mathrm{a} 000487$

ESM 1. Tables (.xlsx)

Systematic review of empirical EJPA papers performing measurement invariance texts.

\section{References}

Bauer, D. J. (2017). A more general model for testing measurement invariance and differential item functioning. Psychological Methods, 22, 507-526. https://doi.org/10.1037/met0000077

Brandmaier, A. M., von Oertzen, T., McArdle, J. J., \& Lindenberger, U. (2013). Structural equation model trees. Psychological Methods, 18, 71-86. https://doi.org/10.1037/a0030001

Greiff, S., \& Heene, M. (2017). Why psychological assessment needs to start worrying about model fit. European Journal of Psychological Assessment, 33, 313-317. https://doi.org/ 10.1027/1015-5759/a000450

Hatlevik, O. E., Scherer, R., \& Christophersen, K.-A. (2017). Moving beyond the study of gender differences: An analysis of 
measurement invariance and differential item functioning of an ICT literacy scale. Computers \& Education, 113, 280-293. https://doi.org/10.1016/j.compedu.2017.06.003

Hildebrandt, A., Lüdtke, O., Robitzsch, A., Sommer, C., \& Wilhelm, O. (2016). Exploring factor model parameters across continuous variables with local structural equation models. Multivariate Behavioral Research, 51, 257-278. https://doi.org/10.1080/ 00273171.2016 .1142856

Kane, M. T. (2013). Validating the interpretations and uses of test scores. Journal of Educational Measurement, 50, 1-73. https://doi.org/10.1111/jedm.12000

Khojasteh, J., \& Lo, W.-J. (2015). Investigating the sensitivity of Goodness-of-Fit indices to detect measurement invariance in a bifactor model. Structural Equation Modeling: A Multidisciplinary Journal, 22, 531-541. https://doi.org/10.1080/10705511. 2014.937791

Kim, E. S., Cao, C., Wang, Y., \& Nguyen, D. T. (2017). Measurement invariance testing with many groups: A comparison of five approaches. Structural Equation Modeling: A Multidisciplinary Journal, 24, 524-544. https://doi.org/10.1080/10705511.2017. 1304822

Marsh, H. W., Guo, J., Parker, P. D., Nagengast, B., Asparouhov, T., Muthen, B., \& Dicke, T. (2017). What to do when scalar invariance fails: The extended alignment method for multigroup factor analysis comparison of latent means across many groups. Psychological Methods. https://doi.org/10.1037/ met0000113

Marsh, H. W., Morin, A. J., Parker, P. D., \& Kaur, G. (2014). Exploratory structural equation modeling: An integration of the best features of exploratory and confirmatory factor analysis. Annual Review of Clinical Psychology, 10, 85-110. https://doi.org/10.1146/annurev-clinpsy-032813-153700

Marsh, H. W., Muthén, B., Asparouhov, T., Lüdtke, O., Robitzsch, A., Morin, A. J. S., \& Trautwein, U. (2009). Exploratory structural equation modeling, integrating CFA and EFA: Application to students' evaluations of university teaching. Structural Equation Modeling: A Multidisciplinary Journal, 16, 439-476. https:// doi.org/10.1080/10705510903008220

Meredith, W. (1993). Measurement invariance, factor analysis and factorial invariance. Psychometrika, 58, 525-543. https://doi. org/10.1007/bf02294825
Muthén, B., \& Asparouhov, T. (2017). Recent methods for the study of measurement invariance with many groups: Alignment and random effects. Sociological Methods \& Research. https:// doi.org/10.1177/0049124117701488

Pendergast, L. L., von der Embse, N., Kilgus, S. P., \& Eklund, K. R. (2017). Measurement equivalence: A non-technical primer on categorical multi-group confirmatory factor analysis in school psychology. Journal of School Psychology, 60, 65-82. https:// doi.org/10.1016/j.jsp.2016.11.002

Rutkowski, L., \& Svetina, D. (2014). Assessing the hypothesis of measurement invariance in the context of large-scale international surveys. Educational and Psychological Measurement, 74, 31-57. https://doi.org/10.1177/0013164413498257

Van De Schoot, R., Kluytmans, A., Tummers, L., Lugtig, P., Hox, J., \& Muthen, B. (2013). Facing off with choosing between Scylla and Charybdis: A comparison of scalar, partial, and the novel possibility of approximate measurement invariance. Frontiers in Psychology, 4(730). https://doi.org/10.3389/fpsyg.2013.00770

Woods, C. M. (2009). Evaluation of MIMIC-model methods for DIF testing with comparison to two-group analysis. Multivariate Behavioral Research, 44, 1-27. https://doi.org/10.1080/ 00273170802620121

\section{Samuel Greiff}

Institute of Cognitive Science and Assessment (COSA)

University of Luxembourg

11, Porte des Sciences

4366 Esch sur Alzette

Luxembourg

samuel.greiff@uni.lu

\section{Ronny Scherer}

Department of Teacher Education and School Research (ILS)

Faculty of Educational Sciences

University of Oslo

Postbox 1099 Blindern

0317 Oslo

Norway

ronny.scherer@cemo.uio.no 